\title{
Psychophysiological stress response in SCUBA divers: The contribution of negative automatic thoughts and negative emotions
}

\author{
Mirela Zec ${ }^{1,2} \cdot$ Vesna Antičević $^{2}$ (D) $\cdot$ Linda Lušić Kalcina $^{3}$ (D) Zoran Valić $^{4}$ (D) Joško Božić ${ }^{5}$ (D)
}

Accepted: 11 February 2022

(c) The Author(s) 2022

\begin{abstract}
The research questions in this study were to examine the contribution of negative automatic thoughts and negative emotions, measured by two newly-constructed questionnaires, to psychophysiological stress responses among more experienced military and less experienced recreational divers. Further, we examined whether daily variations in cortisol levels could be attributed to the impact of negative thoughts/emotions rather than diving experience. Altogether 15 recreational and 14 military male divers participated in the dive wherein stress responses were measured by anxiety measures (DASS-21 and STAI) and cortisol salivary concentrations. The results of factor analysis and principal component analysis demonstrated acceptable construct validity and internal consistency of both questionanires. Recreational and military divers did not differ significantly in the proportion of negative thoughts and emotions, while recreational divers had significantly higher levels of cortisol release after awakening and immediately before/after diving, indicating their more intense stress responses. A significant interaction between daily variations in cortisol secretion and negative emotions indicated a greater importance of emotions in response to diving stress rather than diving experience. Accordingly, using a multiple regression analysis showed that more pre-diving negative emotions predicted higher levels of pre-diving anxiety and depression as well as more cortisol release. More negative thoughts predicted higher levels of depressive symptoms, while belonging to a group of recreational divers was a significant predictor of higher pre-diving anxiety. This study points to the importance of considering cognitive and emotional experiences, particularly in recreational divers, for the purpose of prevention of negative psychophysiological stress responses prior to diving.
\end{abstract}

Keywords Pre-diving automatic thoughts $\cdot$ Emotions $\cdot$ Cortisol $\cdot$ anxiety $\cdot$ Stress $\cdot$ Validation

\section{Introduction}

SCUBA diving poses a challenge for psychophysiological adaptation to an underwater environment. Much effort is being invested into increasing the safety of diving and preventing diving accidents that may occur due to exposure to diving-related stressors. Diving is considered a stressful activity caused by exposure to cumulative structural (exposure to high pressures and aquatic environment), environmental (variations of temperature, thermal conductivity and visibility), physiological (toxicity of breathing gases) and psychological (changes in anxiety and sensory stimulation) stressors (Colodro Plaza et al., 2014) where 50\% of divers experience an occurrence of stress-induced anxiety, panic,

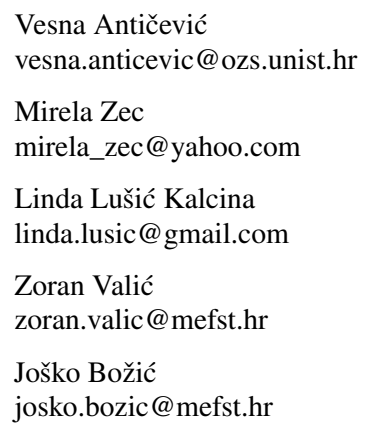

1 Department of Transfusion Medicine, Split University Hospital, Split, Croatia

2 Department of Health Studies, University of Split, Split, Croatia

3 School of Medicine, Department of Neuroscience, University of Split, Split, Croatia

4 School of Medicine, Department of Physiology, University of Split, Split, Croatia

5 School of Medicine, Department of Pathophysiology, University of Split, Split, Croatia 
or phobia while diving (Bachrach \& Egstrom, 1987; Morgan, 1985). SCUBA divers who find themselves under acute stress and experiencing high anxiety struggle to cope with not only underwater circumstances but also the psychophysiological effects of their stress response (Imber, 2018).

\section{Psychophysiological Stress Responses and Emotional Dysregulation during Diving}

At the psychological level, diving-related stressors may elicit emotional responses (Bonnet et al., 2008) leading to the dysregulation of emotions, including the initiation, the inhibition or modulation of the internal emotional states, emotion-related cognitions, physiological processes and behaviors (Siegler et al., 2006). Cognitive strategies, such as catastrophizing, rumination and worry can become maladaptive and elicit negative stress responses, including anxiety and depressive symptoms. The cognitive models of anxiety and depression (Beck, 1980; Beck, 1991; Clark, 1986; Clark \& Beck, 2011) emphasizes the disturbing influence of cognitive processes (e.g., difficulty in processing negative information) manifested through negative automatic threat-relevant thoughts (NAT's) affecting emotion regulation strategies and neurobiological mechanisms (e.g., HPA axis overactivation and higher rates of cortisol production) (Compare et al., 2014). The contribution of the NAT's and negative emotions before and during diving has not been investigated so far. In the context of diving activities NAT's can appear as a result of diving-related stressors (e.g., possible equipment problems, diving difficulty equalizing ears, fatigue of overexertion, muscle cramps, chest tightness, hyperventilation, inhaled water instead of air, motion sickness, fear of unknown, task overload) (Colvard, 2000) and trigger anxiety at the emotional (fear, helplessness, inability to control) and physiological (hyperventilation, palpitations, pounding heart, accelerated pulse rate, a sensation of shortness of breath, or a smoothening feeling) levels, starting before diving and can be intensified during a dive.

At the physiological level, responses to diving stressors are known to be associated with activation of the autonomous nervous system (ANS) and the hypothalamus-pituitary-adrenal (HPA) axis hormones, including increased adrenocorticotropin (ACTH) and cortisol levels related to immersion depth (Anegg et al., 2002; Marlinge et al., 2019; Zarezadeh \& Azarbayjani, 2014). In addition, changes in ACTH and cortisol release can also be a result of divingrelated stress and anxiety caused by reduced perception of control over diving outcomes because of unpredictable dive conditions and lack of access to behavioral responses that can control outcomes (Dickerson \& Kemeny, 2004; Biersner et al., 1981). Although it is difficult to determine exactly which proportion of the variance of diving stress response can be attributed to diving conditions and which to psychological distress, there is a scientific consensus that the diving-related hormonal imbalances can be the result of the combined effects of both factors. Accordingly, in regard to Clark \& Beck's model (Clark \& Beck, 2011), original basal state in combination with individual reactivity to stress may determine which brain circuits are engaged; then, the individual's ability to moderate the degree of reactivity or dampen the thought after engagement will determine overall stress level. Therein circuit integrity and prior psychological status comes into play by giving an estimate of the degree by which the infralimbic cortex can dampen the ANS and hippocampus can dampen the HPA response.

For measuring NAT's and negative emotions in divers two questionnaires have been developed for the purpose of this study. The construct of NAT's was originally developed within the framework of Beck's cognitive theory of depression (Beck, 1980) where Beck describes automatic thoughts as a form of inner speech. When these thoughts are negative, they become crucial for the appearance of various mental states such as depression and anxiety. Taking into account the specificity of diving populations in this study, we developed The pre-diving automatic thoughts questionnaire where the statements were compiled based on a study of literature sources on diving anxiety and the most frequently cited diving problems, as suggested by Colvard (2000), that can result from diving. For measuring diving-related emotions, The positive and negative emotions scale was constructed, according to theoretical thesis about two-dimensional distribution of emotions in which one dimension represents "Arousal" level and the second one represents "Valence", positive or negative, of that emotion (Munoz-de-Escalona \& Canas, 2017). Thus, for example, "fear" would be an emotion with a high level of arousal and a negative valence, while "pleasure" would be a low-activation positive-valence emotion. The psychometric properties of both measures have been reported.

Furthermore, it needs to be emphasized that psychophysiological changes appearing in the diver's body during diving are the result of mutual influence of individual and situation-specific factors, including cognitive assessment and emotional regulation associated with the perception of risk, together with type of diving and diving experience that also could moderate the threshold of psychological resilience in water.

\section{The Contribution of Type of Diving and Diving Experience to the Psychophysiological Stress Response}

SCUBA diving may be done recreationally or professionally, including military, scientific or public safety roles. Although both recreational and military divers are subject to the same immutable laws of physics, have the same 
physiological limitations and operate in the same non-respirable medium and hyperbaric conditions, they often differ in diving motives and goals, risk acceptance levels, length and rigor of diving training, physical fitness (Blumenberg, 1996) as well as in psychological characteristics, which can affect experiencing diving stress caused by situational factors (water environment), and the personality dispositions, including anxiety, mood states and physiological responses.

Compared to recreational divers, military divers have found to be less assertive, displayed higher superego scores and more practical orientation, shrewdness, group orientation and self-sentiment (van Wijk, 2002). Prevalence of common mood and anxiety conditions among military divers appeared to reflect local general population estimates (van Wijk et al., 2020).

In the recent study (St Leger Dowse et al., 2019), it was found that recreational divers revealed rates and severity of depression and anxiety comparable to, or marginally lower, than national surveys although feeling of anxiety and panic before or during a dive was found in a large number of recreational SCUBA divers. It is not surprising since recreational diving, usually practiced for sport purposes, is mostly based on self-responsibility and short training, and therefore risk awareness and personal characteristics of individuals are still decisive factors for the occurrence of diving accidents (Lucrezi et al., 2018).

The current knowledge on the effects of trait anxiety and its emotional and physiological correlates on the behavior of divers is already well documented in world literature indicating trait anxiety as a reliable predictor of experiencing panic during diving, especially in military divers (Biersner \& Larocco, 1987; van Wijk, 2002, 2018), while the psychological mechanisms of anxiety and panic are not sufficiently highlighted to recreational divers (Walton, 2018). In general, the research findings indicate that both military (Biersner \& LaRocco, 1983; Morgan, 1995; van Wijk, 2014, 2018) and recreational (Heyman et al., 1980; Niewiedział et al., 2019) divers have low scores on measures of trait anxiety, with only moderate increase in state anxiety before diving in beginning SCUBA students (Griffiths et al., 1978).

Only a few studies have examined state anxiety that may be triggered by internal (e.g., NAT's and negative emotions) or external (situational) factors related to diving. In the study of Niewiedział et al. (2019) it was found that the divers, during an exposure in a hyperbaric chamber at a depth of 30 and $60 \mathrm{~m}$, experienced state anxiety at a low level, with some divers having a high level of anxiety as a state that most people (Colodro Plaza et al., 2014) experience after decompression from a depth of $60 \mathrm{~m}$.

In another study (Bielec et al., 2006), 90\% of recreational divers presented low and moderate level of trait anxiety (examined during office meeting), but almost half of them felt a strong state of anxiety immediately before the dive whereby they received false information about the presence of strong currents and the appearance of sharks. One possible explanation for these results could be that the perception of the dangers of diving conditions, as suggested by Colvard (2000), has raised anxiety, by provoking NAT's and negative emotions, regardless of low anxiety as a stable personality trait.

No research has been found in which anxiety levels of military and recreational divers have been compared according to diving experience (e.g., total or annual dives or years of diving), but higher stress scores were found in military divers with fewer years of diving experience (Biersner \& LaRocco, 1987). Aditionally, previous research indicates that the more-experienced athletes reported lower intensity levels of reported anxiety symptoms (Hammermeister \& Burton, 1995; Mellalieu et al., 2004), lower worry intensity and more facilitative interpretations of somatic anxiety (Hanton et al., 2008) compared to the less-experienced athletes, due to to the use of more appropriate coping mechanisms. Therefore, in this research, we were interested in whether more experienced military divers had a lower number of NAT's and negative emotions before diving than less experienced recreational divers, which may result in lower levels of anxiety and depression and less pronounced physiological reactivity.

Based on the previous findings presented in the introductory part, the main research questions in this study were to examine the contribution of negative automatic thoughts and negative emotions, measured by two newly-constructed questionnaires, to psychophysiological stress responses among more experienced military and less experienced recreational divers. It was also examined whether daily variations in cortisol levels could be attributed to the impact of negative thoughts/emotions rather than military or recreational diving experience.

Accordingly, the reserch aims have been defined as follows:

- To determine the psychometric properties of the two newly-constructed questionnaires (The positive and negative emotions scale and The pre-diving automatic thoughts questionnaire);

- To examine whether more experienced military divers differ from less experienced recreational divers in cognitive (percentage of pre-diving negative thoughts), emotional (percentage of negative emotions) and physiological (cortisol release) stress responses;

- To determine whether variations in cortisol levels on the day of the dive can be attributed to psychological factors (NAT's and negative emotions) rather than military or recreational diving experience;

- To examine whether pre-diving NAT's and negative emotions as well as belonging to a group of military/recrea- 
tional divers can alter both endogenous levels of stress hormones (cortisol release) and the levels of anxiety and depressive symptoms.

Based on the previous findings, it was expected to find more NAT's, negative emotions as well as higher cortisol levels immediately before the dive in the group of the less experienced recreational divers than in the group of more experienced military divers, according to earlier findings indicating higher anxiety in groups of less experienced military divers (Biersner \& LaRocco, 1987) and similar professions (e.g., athletes) (Hammermeister \& Burton, 1995; Mellalieu et al., 2004). Further, based on previous knowledge (Bachrach \& Egstrom, 1987; Colodro Plaza et al., 2014; Morgan, 1985), it was expected that daily variations in cortisol levels will be attributed to pre-diving NAT's and negative emotions rather than to diving experience. Finally, in line with well-recognized theoretical approaches (Beck, 1980; Beck, 1991; Clark, 1986; Clark \& Beck, 2011) and earlier empirical finding indicating higher level of state anxiety immediately before the dive (Bielec et al., 2006), it was expected that more NAT's and negative emotions would predict higher levels of anxiety and depression, as well as, higher cortisol levels before the dive (Dickerson \& Kemeny, 2004; Biersner et al., 1981).

\section{Materials and Methods}

\section{Participants}

Participation in the study included the voluntary consent of participants for a one-time SCUBA dive and was offered to the diving club for recreational diving and to the Croatian military diving training department. After interested divers expressed their consent for participation to their superiors, a convenience sample of a total of 29 volunteers was finally assembled, of which 14 were military divers and 15 recreational SCUBA divers. All participants were male, aged from 32 to 51 years. The exclusion criteria were diseases and psychophysical states that are known to influence HPA axis, psychiatric disturbances and drugs taking that can interfere with physiological condition.

The group of military divers consisted of employees of the Diving Training Department of the Ministry of Defense of the Republic of Croatia. Participants in this group had at least 10 years of diving experience and minimum of 80 dives a year at a depth of $30 \mathrm{~m}$. They completed professional diving education lasting $100 \mathrm{~h}$ of theoretical instruction followed by intensive diving practice course and continuous professional development throughout their working life.
In the group of the recreational divers, the participants had 4-10 years of diving experience and had completed a diving course that included $40 \mathrm{~h}$ of theoretical instruction and a minimum of 20 dives at a depth of $30 \mathrm{~m}$. The groups did not differ considering age $(\mathrm{U}=-.94, \mathrm{p}>0.05)$, body weight $(\mathrm{kg})(\mathrm{U}=.37, p>0.05)$, body height $(\mathrm{cm})$ $(\mathrm{U}=-0.55, p>0.05)$ and $\mathrm{BMI}(\mathrm{U}=-.68, p>0.05)$.

\section{Procedure}

The day before the dive, all subjects were provided with Informed Consent and were instructed how to collect five salivary cortisol samples. They were also instructed not to take cigarettes or alcohol and to plan waking up at 7 a.m. at the day of the dive.

On the day of the dive, all participants woke up around $7 \mathrm{a} . \mathrm{m}$. and slept an average of $7.9 \mathrm{~h}$ the night before. They also reported that they have not been exposed to any extraordinary stressful experiences in previous 7 days that could affect their current psycho-physiological state.

Immediately before and after the dive, all participants completed questionnaires examining current mental state, pre-diving automatic thoughts and emotions that preceded and followed the dive. For the purposes of this study, the subjects from both groups dived at depths of up to $30 \mathrm{~m}$ at an optimal sea temperature of $15^{0}-16^{0} \mathrm{C}$. The dives took place in groups during October 2019. The dive began at $10 \mathrm{a} . \mathrm{m}$. for both groups, and lasted for $50 \mathrm{~min}$, under the already pre-arranged diving regime. Before, during and after the dive, there were not any unexpected events, which could negatively affect research results.

The Ethics Committee of the University of Split, School of Medicine approved this research.

\section{Measures}

Cortisol measurement - Saliva samples were collected at five time points according to a defined protocol (between 7 and 8 a.m. (P1); about 9 a.m. (P2); immediately before the dive (P3); immediately after the dive (P4) and 60 min after the dive (P5). The subjects stored saliva samples in laboratory micro tubes. Saliva samples were stored in the transport medical fridge, within the controlled conditions, until the final storage in the medical fridge at the temperature of $-20{ }^{\circ} \mathrm{C}$ until analysis day. On the day of testing, the samples were dissolved at room temperature, centrifuged for $2 \mathrm{~min}$ / $1400 \mathrm{rpm}$ in NF 048 microcentrifuge. Total of $200 \mu \mathrm{l}$ of saliva supernatant was used for analysis. The measurement of salivary cortisol was processed by ELISA kit (Cortisol kit, Roche Diagnostics USA). An immunoassay analyzer was used for the quantitative and qualitative biochemical tests. The reference intervals were $3.7-30.7 \mathrm{nmol} / 1$. 
The samples were analyzed by two independent medical laboratory diagnosticians who were not familiar with the research groups, neither the time points which samples belonged to. Samples were analyzed based on the manufacturer guidelines, in accordance with the right lab practice.

Depression Anxiety and Stress Scale (DASS (Lovibond $\&$ Lovibond, 1995). was used to measure levels of anxiety, depression and stress symptoms immediately before and after the dive. It consists of 42 items and three subscales measuring depression (e.g., ,I can't seem to experience any positive feeling at all.“), anxiety (e.g., ,I worry about situations in which I might panic and make a fool of myself.") and stress (e.g., ,I find it difficult to relax.)“. The responds are influenced by situational factors and reflect the current circumstances of the respondent. Respondents answered by circling the corresponding number on a four-point Likerttype scale, from 0 - not at all refers to me, to 3 - completely refers to me. The total score is formed as a linear combination of subscale scores. This study used a validated Croatian scale that confirmed the psychometric properties of the original questionnaire and determined the following internal reliability coefficients: .85 for the depression subscale, .83 for the anxiety subscale, and .87 for the stress subscale (Selection, 2019).

The State-Trait Anxiety Inventory (STAI) (Spielberger et al., 1983) is a commonly used measure of trait and state anxiety, consisting of 20 items for assessing trait anxiety and 20 for state anxiety. State anxiety items include: "I am tense; I am worried" and "I feel calm; I feel secure." Trait anxiety items include: "I worry too much over something that really doesn't matter" and "I am content; I am a steady person." All items are rated on a 4-point scale (e.g., from "Almost Never" to "Almost Always"). Higher scores indicate greater anxiety. Internal consistency coefficients for the scale have ranged from .86 to .95 ; test-retest reliability coefficients have ranged from .65 to .75 over a 2-month interval (Spielberger et al., 1983). Test-retest coefficients for this measure in the present study ranged from .69 to .89. Considerable evidence attests to the construct and concurrent validity of the scale (Spielberger, 1989). Only the state anxiety scores were used in this study indicating measuring the psychological and physiological transient reactions directly related to adverse situations in a specific moment.

For measuring both pre and post-diving emotions The positive and negative emotions scale was constructed, consisting of seven positive (adjectives used to describe happiness, motivation, ecstasy, fun, enthusiasm, pleasure and passion) and seven negative (adjectives used to describe selfdisappointment, fear, tension, worry, shame, discomfort and distress) emotions, which can be triggered by an elevated physiological arousal on a situational stimulus, as suggested by Munoz-de-Escalona and Canas (2017). The construction of the scale was performed as follows (Fig. 1).
The participants were asked to assess their current emotional state immediately before and after the dive on a scale ranging from $0 \%$ to $100 \%$. The total score of each respondent represents the average percentage of positive or negative emotions, with the maximum score being $100 \%$ in both cases. Scree plots were examined and enabled a two-factor model solution for Positive and Negative emotions subscale, as shown in Table 3. For the Positive emotions subscale, the first factor (Satisfaction) explained $48.84 \%$ of the variance and the second factor (Excitement) explained $18.46 \%$ of the variance. Comparably, the first factor (Fear) explained $73.67 \%$ of the variance and the second factor (Anxiety and stress) explained $16.25 \%$ of the variance for the Negative emotion subscale (Tables 1 and 3).

A good reliability of the Positive emotion subscale was shown, with a Cronbach's alpha of .76, as well as the reliability of the Negative emotion subscale considering a Cronbach's alpha of .79 (Table 1).

Only the total scores of negative emotions were used in the analysis following theoretical approach used in this study Beck, 1980; Clark \& Beck, 2011) where NAT's can provoke negative emotions leading to an increase in anxiety and depression.

The pre-diving automatic thoughts questionnaire has been constructed to measure the frequency of NAT's before the dive according to the following procedure (Fig. 2). After reviewing scientific literature including Beck' theory of depression, Beck Depression Inventory-BDI and lists of the most common diving-related stressors (e.g., dangerous dive conditions, possible equipment problems and physical/psychological issues, as suggested by Colvard, 2000), a larger number of items for measuring individual thoughts before diving was created. Then, a small pretest of the questionnaire was carrying out, using a small subset of target respondents consisting of three experienced divers who have had previously practical experience as a members of diving clubs. They were asked to recall their own dysphoric experiences before diving and to report associated cognitions, which were added to existing items. Such a list of items was then reconciled in a focus group between a psychologist and three divers. After that, face validity of the items was assessed on three levels: content (undoubted "belonging" to the construct of pre-diving NAT's), measurement (whether it is possible and justified to measure it by divers self-assessment) and verbal (use of vocabulary and, if necessary, jargon used by divers). Next, all items were given for reading to a small group of divers in the diving club who were asked to assess only the comprehensibility of the items, as well as their applicability to the diving context. Items which were assessed as less understandable by any diver were reconsidered or omitted. In that way, a larger number of items were created, which served as a possible "pool" of items from which a new questionnaire was later generated. 


\section{REVIEW OF SCIENTIFIC LITERATURE}

Munoz-de-Escalona, E., \& Canas, J. J. (2017)

\section{EMOTION LIST CONSTRUCTION}

Seven negative (adjectives used to describe self- Seven positive emotions (adjectives used to describe disappointment, fear, tension, worry, shame, discomfort happiness, motivation, ecstasy, fun, enthusiasm, and distress) emotions pleasure and passion)

\section{A PRETEST OF THE QUESTIONNAIRE}

\section{ALL ITEMS INCLUDED IN THE FINAL VERSION}

\section{SELECTION OF CONSTRUCT VALIDITY MEASURES}

DASS-21

STAI

Fig. 1 Construction of The positive and negative emotions scale

Table 1 Reliability measures of The positive and negative emotions scale

\begin{tabular}{llllll}
\hline Positive emotions & $\begin{array}{l}\text { Item-total cor- } \\
\text { relation }\end{array}$ & $\begin{array}{l}\text { Cronbach's } \alpha \text { if } \\
\text { item deleted }\end{array}$ & Negative emotions & $\begin{array}{l}\text { Item-total cor- } \\
\text { relation }\end{array}$ & $\begin{array}{l}\text { Cronbach's } \\
\alpha \text { if item } \\
\text { deleted }\end{array}$ \\
\hline E1 Happy & .70 & .73 & E2 Dissapoiinted & .83 & .76 \\
E5 Motivated & .50 & .74 & E3 Afraid & .88 & .87 \\
E6 Excited & .45 & .74 & E4 Anxious & .54 & .84 \\
E7 Amused & .70 & .73 & E10 Worried & E11 Afraid & .82 \\
E9 Enthusiastic & .72 & .70 & E14 Uncomfortable & .92 & .78 \\
E12 Passionate & .31 & .75 & Negative emotions sum score & 1.000 \\
E13 Content & .83 & .71 & & .79 \\
Positive emotions sum score & 1.000 & .76 & & .79 \\
\hline
\end{tabular}

Finally, the initial screening and selection of items for the compilation of the questionnaire for the implementation of the research was performed. In parallel with the process of making the items of the new questionnaire, the selection of instruments for the validation of the object of measurement was made (Depression, Anxiety and Stress Scale -DASS-21 (Lovibond \& Lovibond, 1995) and Spielberger Trait-Anxiety
Inventory-STAI, Spielberger, 1989). The construction of the questionnaire is presented in Fig. 2.

In this way, a list of 24 thoughts was obtained, the frequency of which was estimated by 29 divers who participated in this study. Respondents had to mark thoughts that went through their minds immediately before the dive. Scoring mode was binary: 0 (,I have no such thought“") and 


\section{REVIEW OF SCIENTIFIC LITERATURE}

\begin{tabular}{|c|c|c|c|c|}
\hline $\begin{array}{l}\text { Beck' Theory Of } \\
\text { Depression }\end{array}$ & $\begin{array}{l}\text { Beck Depression } \\
\text { Inventory }\end{array}$ & $\begin{array}{l}\text { List of dangerous } \\
\text { dive conditions }\end{array}$ & $\begin{array}{l}\text { List of possible } \\
\text { equipment } \\
\text { problems }\end{array}$ & $\begin{array}{c}\text { List of } \\
\text { physical/psycholog } \\
\text { ical issues } \\
\text { suggested by }\end{array}$ \\
\hline & & & & Colvard (2000) \\
\hline
\end{tabular}

\section{A PRETEST OF THE QUESTIONNAIRE}

Divers recalling dysphoric experiences before diving Divers reporting associated cognitions

\section{ADDITIONAL ITEMS CREATED}

FOCUS GROUP

FACE VALIDITY ASSESSMENT

Content

Measurement

Verbal (use of vocabulary and jargon)

ASSESSING COMPREHENSIBILITY AND APPLICABILITY TO THE DIVING CONTEXT

\section{OMITTING ITEMS}

FINAL VERSION OF THE PRE-DIVING AUTOMATIC THOUGHTS QUESTIONNAIRE

SELECTION OF CONSTRUCT VALIDITY MEASURES

DASS-21

STAI

Fig. 2 Construction of The pre-diving automatic thoughts questionnaire 
1 („The thought is present"). The total score is expressed as the sum of positive responses, with a possible range of scores $0-24$.

A categorical principal component analysis (CATPCA) of all items revealed that the questionnaire consists of three recognized factors, which we named Diving fatalities, Panic Attack and Uncertainty explaining a total of $61.78 \%$ of variance. Two items were excluded from the questionnaire since their factor loadings were $<.40$. The final questionnaire contains 22 NAT's (Table 4). The following internal reliability coefficients were established: .91 for the diving fatalities subscale, .78 for the panic attack subscale, and .54 for the uncertainty subscale.

General data questionnaire included information about age, anthropometric characteristics, years of diving experience, exposure to extraordinary stressors in previous 7 days, the information on hours of sleep the night before the dive and waking time on the day of the dive.

\section{Statistical Analyses}

Data were recoded, sorted, and prepared for analysis using the SPSS version 25.0 software package. There was no missing data in the dataset. Descriptive statistical procedures (means, standard deviations and percentages) were used to describe the number of NAT's and negative emotions.

We conducted a reliability analysis for the internal consistency using the Cronbach's alpha coefficient. If a scale was assessed as reliable, explaratory (EFA) factor analysis were performed to determine the factor structure. Sums of Squared percentage of variance were calculated, and cumulative percentage of variance. Additionally, a Kaiser-MeyerOlkin Measure of Sampling Adequacy was calulcated, as well as Bartlett's test of sphericity. A Varimax rotation was performed in order to assess factor component loadings. Factor analysis using Varimax rotation was performed in order to examine factors derived from Positive and negative emotions scale. Additionally, cofirmatory factor analysis was performed in order to assess the model fit summary of positive and negative emotions scale with identified factors. The comparative fit index (CFI) was used to analyse the model fit by examining the discrepancy between the data and the hypothesized model. In The pre-diving automatic thoughts questionnaire, considering that NAT's questionnaire included only questions with binary answers, a categorical principal component analysis (CATPCA) was performed.

Convergent validity was established based on the correlation of the investigated scales with the assessment often used as the gold-standard for negative emotions such as depression and anxiety, STAI and DASS scales.

Descriptive analysis such as frequency, mean and standard deviation was calculated as per the requirement for data analysis. Student t-test was used to find out the differences between recreational and military divers in daily cortisol variations. In order to investigate changes in cortisol levels from the first (cortisol awakening response - CAR) to the fifth measuring point (one hour after the dive) within both recreational and military diver's groups we used t- test. Next, interaction effects of within-subjects cortisol variations during the day of the dive with NAT's, negative emotions and group affiliation were investigated using Mauchly W test of sphericity. Finally, multivariate regression analyses were conducted with the aim of determining the contributions of group affiliation, NAT's and negative emotions to anxiety and stress, as well as to participants' cortisol levels. The overall significance for regression models was tested by ANOVA tests (F-ratio). $P$-values of less than .05 were considered statistically significant.

\section{Results}

\section{Factor Structure}

The Kaiser-Meyer-Olkin statistic proved satisfying sampling adequacy of the data in the Positive emotions $(\mathrm{KMO}=.77)$ and Negative emotions subscale $(\mathrm{KMO}=.83)$, enabling the factor analysis. The Bartlett's test of sphericity was significant for the Positive emotion subscale $(\times 2(21)=80.8, p<.001)$, and the Negative emotions subscale $(\times 2(21)=225.02, p<.001)$.

A good reliability of the Positive emotion subscale was shown, with a Cronbach's alpha of .76, as well as the reliability of the Negative emotion's subscale considering a Cronbach's alpha of .79 (Table 1).

As presented in Table 2, the correlation of Negative emotions subscale with STAI score $(r=.71)$ and Depression $(r=.75)$, Anxiety $(r=0.86)$ and Stress subscale of DASS $(r=.59)$ demonstrated good validity. Somewhat lower correlation coefficients were established for Positive emotions subscale with Depression $(r=-.38)$ and Anxiety $(r=-.40)$ subscale in DASS, as well as with STAI $(r=-.38)$. Regarding The pre-diving automatic thoughts questionnaire, the convergent validity was satisfying considering a positive correlation of sum scores of the aforementioned questionnaire: wirh DASS Depression subscale $(r=.71)$, Anxiety subscale $(r=.63)$ and Stress subscale $(r=.62)$, as well as with STAI sum score $(r=.50)$.

Scree plots were examined and enabled a two-factor model solution for Positive and Negative emotions subscale, as shown in Table 3. For the Positive emotion subscale, the first factor explained $48.84 \%$ of the variance and the second factor explained $18.46 \%$ of the variance. Comparably, the first factor explained $73.67 \%$ of the variance and the second factor explained $16.25 \%$ of the variance for the Negative emotion subscale. 
Table 2 Convergent validity of the The pre-diving automatic thoughts questionnaire and The positive and negative emotions scale

\begin{tabular}{|c|c|c|c|c|c|c|c|}
\hline & \multirow[t]{2}{*}{ NAT's } & \multicolumn{3}{|l|}{ Negative emotions } & \multicolumn{3}{|l|}{ Positive emotions } \\
\hline & & $\begin{array}{l}\text { Negative emotions } \\
\text { sum score }\end{array}$ & $\begin{array}{l}\text { Factor } 1 \\
\text { Fear }\end{array}$ & $\begin{array}{l}\text { Factor } 2 \\
\text { Anxiety and } \\
\text { stress }\end{array}$ & $\begin{array}{l}\text { Positive emotions } \\
\text { sum score }\end{array}$ & $\begin{array}{l}\text { Factor } 1 \\
\text { Satisfaction }\end{array}$ & $\begin{array}{l}\text { Factor } 2 \\
\text { Excite- } \\
\text { ment }\end{array}$ \\
\hline Diving NAT & - & $.68^{*}$ & $.50 \dagger$ & $.47 \ddagger$ & -0.06 & -.23 & .17 \\
\hline DASS Depression & $.71^{*}$ & $.75^{*}$ & $.64^{*}$ & $.39 \ddagger$ & $-.38 \ddagger$ & $-.52 \dagger$ & -.02 \\
\hline DASS Anxiety & $.63^{*}$ & $.86^{*}$ & $.70^{*}$ & $.49 \dagger$ & $-.40 \ddagger$ & $-.63 *$ & .08 \\
\hline DASS Stress & $.62 *$ & $.59 *$ & .36 & $.50 \dagger$ & -.12 & -.32 & .16 \\
\hline STAI sum score & $.50 \dagger$ & $.71^{*}$ & $.56 \dagger$ & $.43 \ddagger$ & $-.38 \ddagger$ & $-.55 \dagger$ & -.01 \\
\hline Positive emotions sum score & -.06 & $-.39 \ddagger$ & $-.43 \ddagger$ & -.06 & & $.74 *$ & $.66^{*}$ \\
\hline Negative emotions sum score & $.68^{*}$ & - & $.82 *$ & $.58 \dagger$ & $-.39 \ddagger$ & $-.64 *$ & .09 \\
\hline Factor 1 Negative emotions & $.50 \dagger$ & $.82 *$ & - & - & $-.43 \ddagger$ & $-.64 *$ & .05 \\
\hline Factor 2 Negative emotions & $.47 \dagger$ & $.58 \dagger$ & - & - & -.06 & -.19 & .10 \\
\hline Factor 1 Positive emotions & -.23 & $-.64 *$ & $-.64 *$ & -.19 & $.74^{*}$ & - & - \\
\hline Factor 2 Positive emotions & .17 & .09 & .05 & .10 & $.66^{*}$ & - & - \\
\hline
\end{tabular}

Note: Correlation coefficient of The pre-diving automatic thoughts questionnaire and The positive and negative emotions scale with criterion measures (STAI and DASS scores for each sum score and established factors)

${ }^{*} p<.001 ; \dagger p<.01 ; \dagger p<.05$

Table 3 Factor analysis of The positive and negative emotions scale

\begin{tabular}{|c|c|c|c|c|}
\hline & \multicolumn{2}{|c|}{ Positive emotions 2 factor solution } & \multicolumn{2}{|c|}{$\begin{array}{l}\text { Negative emotions } 2 \text { factor } \\
\text { solution }\end{array}$} \\
\hline & $\begin{array}{l}\text { Factor } 1 \\
\text { Satisfaction }\end{array}$ & $\begin{array}{l}\text { Factor } 2 \\
\text { Excitement }\end{array}$ & $\begin{array}{l}\text { Factor } 1 \\
\text { Fear }\end{array}$ & $\begin{array}{l}\text { Factor } 2 \\
\text { Anxiety } \\
\text { and stress }\end{array}$ \\
\hline $\begin{array}{l}\text { Kaiser-Meyer-Olkin Measure of } \\
\text { Sampling Adequacy }\end{array}$ & \multicolumn{2}{|l|}{0.764} & \multicolumn{2}{|l|}{0.825} \\
\hline Bartlett's test of sphericity & \multicolumn{2}{|c|}{$\times 2(21)=80.8, p<.001$} & \multicolumn{2}{|c|}{$\times 2(21)=225.02, p<.001$} \\
\hline Initial Eigenvalues & 3.42 & 1.29 & 5.16 & 1.14 \\
\hline$\%$ of Variance & 48.84 & 18.46 & 73.67 & 16.25 \\
\hline Cumulative \% & 48.84 & 67.30 & 73.67 & 89.92 \\
\hline Rotation Sums of Squared & 2.99 & 1.72 & 4.40 & 1.89 \\
\hline$\%$ of Variance & 42.73 & 24.57 & 62.84 & 27.08 \\
\hline Cumulative \% & 42.73 & 67.30 & 62.84 & 89.92 \\
\hline \multicolumn{5}{|c|}{ Item loadings } \\
\hline E1 & .83 & & & \\
\hline$E 2$ & & & .95 & \\
\hline E3 & & & .81 & \\
\hline E4 & & & & .80 \\
\hline E5 & .86 & & & \\
\hline E6 & & .59 & & \\
\hline E7 & .80 & & & \\
\hline E8 & & & & .97 \\
\hline E9 & & .70 & & \\
\hline E10 & & & .92 & \\
\hline E11 & & & .94 & \\
\hline E12 & & .82 & & \\
\hline$E 13$ & .83 & & & \\
\hline E14 & & & .90 & \\
\hline
\end{tabular}

Note. Total variance explained and factor component loadings for The positive and negative emotions scale factor solutions 
Table 4 Principal component analysis of The pre-

diving automatic thoughts questionnaire

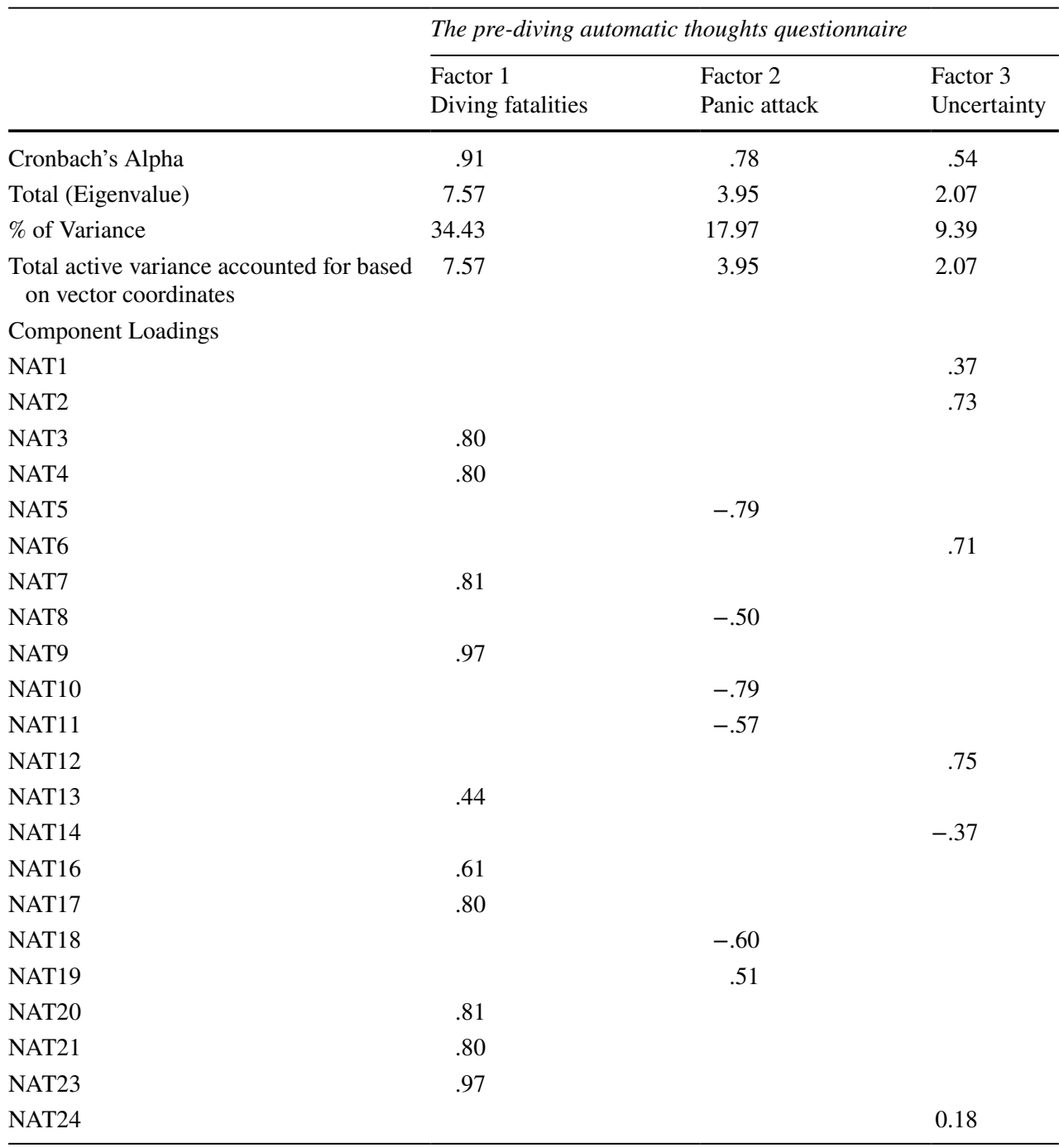

Factor component loadings for The pre-diving automatic thoughts questionnaire factor solutions
As shown in Table 4, following categorical principal component analysis of all items of The pre-diving automatic thoughts questionnaire, we established that the questionnaire consists of three recognized factors, explaining a total of $61.78 \%$ of variance. Following CFA, a comparative fit index (CFI) was acceptable at .92. Considering that CFI values range from 0 to 1 , with larger values indicating better fit, we considered a given value of .92 as an acceptable indicator of the suggested model (Bentler, 1990).

\section{Differences in the Number of NAT's and Negative Emotions As Well as Cortisol Levels between Military (more Experienced) and Recreational (less Experienced) Divers}

The average percentage of the pre-diving NAT's and negative emotions in recreational and military divers are shown in Fig. 3. No statistically significant difference in the

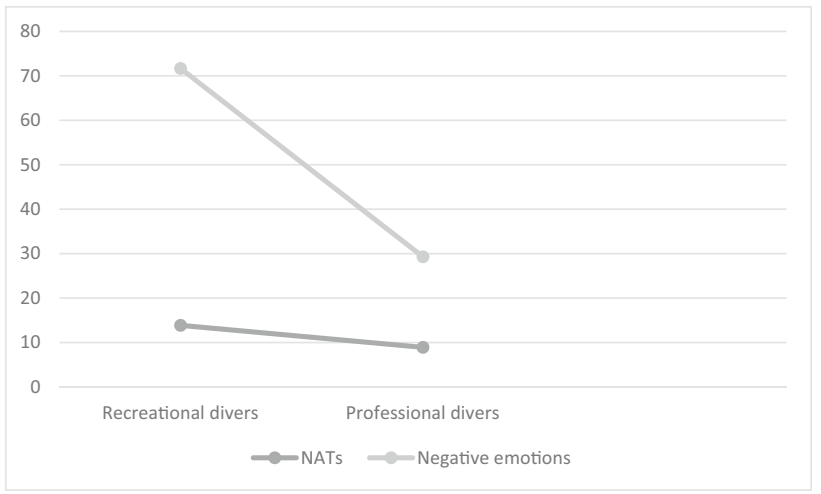

Fig. 3 Percentages of NAT's and negative emotions between recreational and professional divers

number of NAT's was determined between the observed groups $(t=.29, p>.05)$. By observing the differences 
Fig. 4 Differences in cortisol levels within and between groups of professional and recreational divers

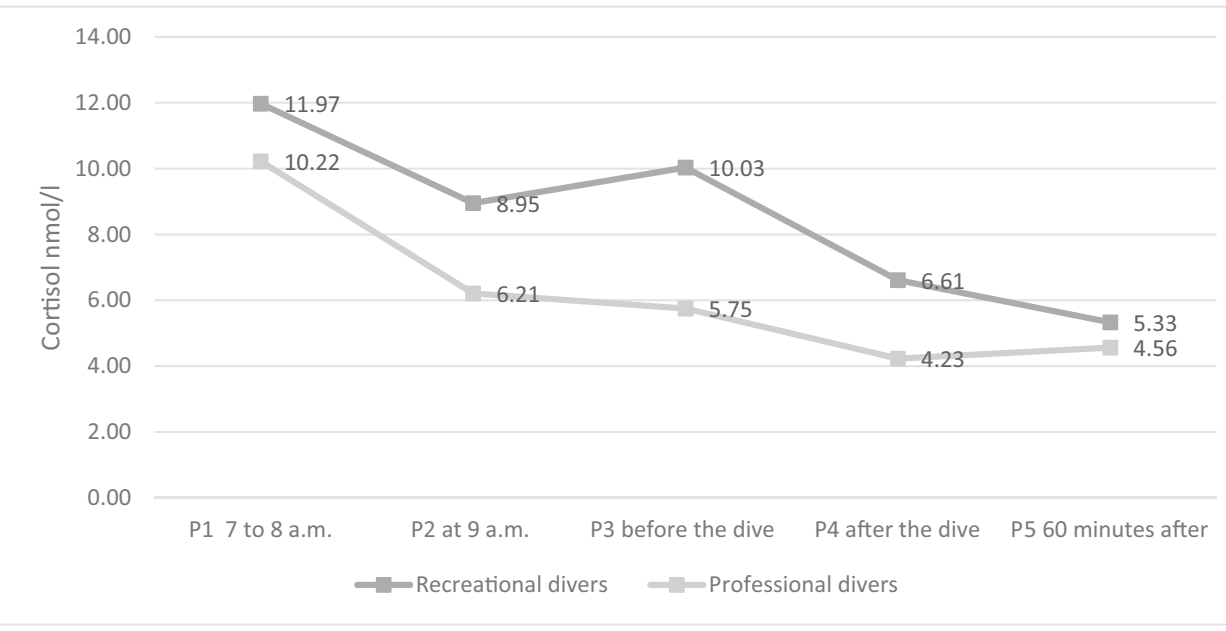

in negative emotions before the dive, no significant differences were established $(t=1.08, p>.05)$. Further, as it can be seen from the Fig. 4, average cortisol values significantly decreased at different daily measurement points within both groups. Recreational divers reported significant higher levels of cortisol measured at 9 a.m. $(t=2.22, p=.036)$; immediately before the dive $(t=2.72$, $p=.012)$ and immediately after the dive $(t=2.17, p=.042)$ compared to the military divers, while in other measurement points the differences between groups were not established.

Univariate analysis of differences in cortisol levels across all 5 time points assessed in the current study revealed a significant effect of cortisol level changes in a within-subject design in both, recreational (Mauchly $W=.14, p=.004 ; F=8.15, p=.001)$ and military divers (Mauchly $W=.12, p=.004 ; F=7.82, p=.003$ ) (Table 5). Pairwise comparisons in recreational divers revealed significant differences among the $\mathrm{P} 1$ and $\mathrm{P} 2(p=.010)$ as well as among P1 and P5 measurement point $(p=.006)$. In addition, levels of cortisol at P2 were significantly different from P5 ( $p=.020)$. Differently, in military divers only time point P1 was significantly different from the cortisol levels measured at P4 $(p=.021)$ and P5 $(p=.030)$.

However, when interaction effects of a diving group and variations in cortisol levels during the day of the dive were assessed, no significant interaction effects were reported, as indicated in Table 5.
Furthermore, given that there is an interaction of variability in cortisol levels during the day of the dive and the level of negative emotions, it can be assumed that variability of cortisol during the day of the dive depends on the negative emotional state but not solely on diving experience because the interaction with the diving group has not been established.

\section{The Role of the NAT's, Negative Emotions and Group Affiliation in Predicting anxiety and Stress As Well as Cortisol Levels before the Dive}

The results of the regression analysis used to determine the predictors of anxiety, depression and stress before diving can be seen in Table 6. NAT's, negative emotions and group affiliation were used as predictor variables, while the measures of anxiety and stress were used as dependent variables.

Regression analyses have shown that group affiliation, NAT's and negative emotions together explained $79 \%$ of the total variation in anxiety measured by DASS and $56 \%$ measured by STAI, whereby negative emotions and affiliation to a group of recreational divers significantly affected anxiety (measured by DASS and STAI) immediately before the dive $(p<0.001)$. This model is significant, with a $F=30.68$, $p<0.001$ (Table 6).

Furthermore, group affiliation, NAT's and negative emotions together explained $63 \%$ of the total variation in depressive symptoms. Also, the model was significant, with
Table 5 Interaction effects of within-subjects cortisol variations during the day of. the dive with NAT's, negative emotions and group affiliation

\begin{tabular}{llc}
\hline Variables & $\mathrm{F}$ & $p$ \\
\hline Variations in cortisol levels during the day of the dive & 8.08 & $<.001^{* *}$ \\
Variations in cortisol levels during the day of the dive*Negative emotions & 3.41 & $.02^{*}$ \\
Variations in cortisol levels during the day of the dive*NAT's & 1.12 & .35 \\
Variations in cortisol levels during the day of the dive*Group affiliation & .79 & .50
\end{tabular}


Table 6 Negative automatic thoughts, negative emotions and group affiliation in predicting negative affectivity and cortisol levels before the dive

\begin{tabular}{clllll}
\hline & & DASS Anxiety & STAI Anxiety & Depression & $\begin{array}{l}\text { Cortisol } \\
(\mathrm{P} 4) \\
\text { nmol/L }\end{array}$ \\
\hline $\mathrm{GA}$ & & & & 0.35 \\
& $\mathrm{R}^{2}$ & 0.79 & .56 & 0.63 & 2.74 \\
& Standard Error & 2.97 & 6.77 & 3.68 & 4.42 \\
& $\mathrm{~F}$ & 30.68 & 13.06 & 16.75 & .01 \\
& $\mathrm{p}$ & $<.001$ & $<.001$ & .001 & -0.27 \\
& $\mathrm{~B}$ & -0.23 & -0.33 & -0.19 & -1.64 \\
& $\mathrm{t}$ & -2.37 & -2.55 & -1.59 & .11 \\
& $\mathrm{p}$ & $.03^{*}$ & $.02^{*}$ & .12 & 0.00 \\
$\mathrm{NAT}$ & $\mathrm{B}$ & 0.08 & -0.003 & 0.35 & 0.02 \\
& $\mathrm{t}$ & 0.60 & -0.02 & 2.19 & .99 \\
& $\mathrm{p}$ & .55 & .984 & $.04 *$ & 0.46 \\
$\mathrm{NE}$ & $\mathrm{p}$ & 0.75 & 0.642 & 0.47 & 2.07 \\
& $\mathrm{t}$ & 5.91 & 3.73 & 2.98 & $.05 *$ \\
\hline
\end{tabular}

Note. GA group affiliation, NAT's negative automatic thoughts, NE negative emotions, $\beta$ standardized beta coefficient, $t$ t-test, $F$ F ratio (ANOVA), $R^{2}$ coefficient of determination

$* p<.05 ; * * p<.01$ a $F=16.75, p<0.001$. Thereby, a larger percentage of both NAT's $(p<.05)$ and negative emotions $(p<.01)$ predict more depressive symptoms before the dive.

As can also be seen in Table 6, the regression analysis predicting cortisol levels immediately before the dive indicated that more negative emotions accounted for a significant proportion $(35 \%)$ of the variance $(p<.05)$. The model is significant, with a $F=4.42, p=0.01$.

\section{Discussion}

The main research questions in this study were to examine the contribution of NAT's and negative emotions, measured by two newly-constructed questionnaires, to psychophysiological stress responses in more experienced military and less experienced recreational divers. It was also examined whether daily variations in cortisol levels could be attributed to the impact of NAT's and negative emotions rather than military or recreational diving experience.

The psychometric properties of both questionnaires The positive and negative emotions scale and The pre-diving automatic thoughts questionnaire were verified using construct validity, and the reliability was tested using Cronbach's alpha reliability. The results of factor analysis and principal component analysis demonstrate acceptable validity and internal consistency (reliability), including acceptable model fit, indicating that both instruments can be reccomended in future statistical and practical studies.

The findings in this study indicated a relatively small amount of pre-diving NAT's and negative emotions in both groups, with no statistically significant differences between them, considering that all participants were healthy experienced divers who completed training programs and developed coping strategies for dealing with diving challenges. Despite the lack of significant differences between the groups, the results indicated more NAT's and negative emotions among recreational (13.87\% for NAT's and $71.67 \%$ for negative emotions) versus military $(8.92 \%$ for NAT's and $29.29 \%$ for negative emotions) divers, indicating greater likelihood of pre-diving NAT's and negative emotions among recreational divers immediately before the dive.

However, regression analyses have shown that NAT's had significant effects in predicting depressive symptoms, suggesting that negative cognition preceding diving can challenge depressive symptoms, such as feelings of worthlessness or guilt, fixating on past failures or self-blame, difficulties in thinking, concentrating, making decisions or remembering that can interfere with underwater functioning. In addition, the findings of this study indicated an association between negative emotions and higher emotional dysregulation, i.e., with more anxiety and depressive symptoms, as well as higher cortisol secretion immediately before diving. These findings are consistent with theoretical approaches emphasizing the importance of cognitive and emotional factors for emotional regulation in activities that are perceived as threatening (Beck, 1991; Siegler et al., 2006). In the context of diving, negative emotional responses to the perceived risk of SCUBA diving can serve as risk factors for divers' safety.

Negative emotions were found to be strongly correlated with anxiety in previous study, indicating that exposure to stress generally can cause dysregulation of emotions, which in turn elicits negative psychological and physical symptoms 
(e.g., anxiety and stress-induced physiological symptoms) (Ng et al., 2019).

This study also showed that recreational divers were more prone to develop higher pre-diving stress responses, whereby belonging to a group of recreational divers predicted more anxiety symptoms, measured by STAI and DASS-21 before the dive. Accordingly, recreational divers had a significantly higher levels of cortisol release at the first measurement point (after awakening), immediately before and after the dive in comparison with military divers. These findings are in accordance with previous studies indicating that observed less vulnerability to diving-related stress in military divers can be the result of both lower anxiety and external factors, such as more diving experience and longer education, that jointly contributed to more efficient stress control during diving (Biersner \& Larocco, 1987; Revelli et al., 2007). Additionally, military divers are commonly very sporty and have a high level of fitness (Blumenberg, 1996). Thus, it can't be excluded that observing differences in the physiological stress response (including the cortisol level) between the diving groups are caused by the likely various psychophysical fitness of the groups.

In this study, serum cortisol levels in five-time intervals on the day of the dive, showed a significant physiological decline within both groups, being the highest after awaking and gradually falling as the day progressed, as the result of cortisol awaking response (CAR). This can be expected since the cortisol level regularly depends on the circadian rhythm with a peak after wakening and all subjects in this study reported waking up at 7 a.m. on the day of the dive. The only increase in the cortisol level was recorded immediately before the dive (although it does not reach the limit of statistical significance) in recreational divers, which may indicate an increase in psychological stress. Considering changes in cortisol levels, as a possible stress indicator during SCUBA diving, research findings have shown mixed results some of them indicating that cortisol increase was evident due to physiological effects of immersion and raised environmental pressure (Zarezadeh \& Azarbayjani, 2014) or hypoxia (Marlinge et al., 2019) rather than anxiety or cold stress. Different results concerning cortisol release could be explained with differences in timing and characteristics of cortisol sampling, differences in submersion process, recent stressors, tobacco use and alcohol consumption as well as differences in professional status and experience of divers. Also, cortisol concentrations could be altered with time of the dive during the day, as it is known that cortisol secretion has certain daily rhythm. Furthermore, cortisol release is susceptible to changes due to all of the mentioned reasons, and therefore it is possible that all these studies have major methodological differences that cause different cortisol readings. In an effort to minimize the impact of possible above-mentioned causes of daily variations in cortisol levels, participants in this study were instructed on nonalcohol and nicotine use and on waking at 7 a.m. on the day of the dive. They also reported about absence of recent stressors in previous days that could interfere with cortisol values on the day of diving. Both groups have dived at the same time, at same diving conditions (sea temperature, diving depth). The significant interaction between daily variations in cortisol secretion on the day of the dive and negative pre-diving emotions suggests that variations in cortisol secretion may be the result of emotional dysregulation rather than diving conditions.

This research has several limitations. The main limitation refers to the small study population that prevents drawing general conclusions about psychological risks before diving. Additionally, we underscore that small study population limited definite conclusions on the factors structure of The prediving automatic thoughts questionnaire assessed with categorical answers, and future studies should provide additional elaboration or confirmation of the component structure recognized in the current study. The use of self-reported data can be the next source of bias. Some participants could be either consciously or unconsciously influenced by "social desirability" while reporting about their diving-related experiences and in that way provided less accurate data. Especially military drivers might be concerned by negative impacts when reporting of anxiety and depressive symptoms as well as negative thoughts and emotions in respect of their job performance.

Regardless to the mentioned limitations, this study provides additional insights on the psychology of diving. The study results point in the direction of the need for early detection of the cognitive (NAT's) and emotional contents (divingrelated negative emotions) that can appear before SCUBA diving and affect diving outcomes. It is recommended that future research continue to study the influence of thoughts and emotions on diving-related stress responses on larger samples in order to better understand the onset and maintenance of anxiety and stress which are known to be some of the most important risk factors for diving-related accidents. Finally, it may be appropriate to determine the the cortisol awakening response -CAR (i.e. the change in cortisol concentration that occurs in the first hour after waking from sleep which is then the highest and gradually decreases during the day) and psychological status on another day when the divers did not have a dive, because it is difficult to make conclusions only on the basis of one sampling on the day the dive took place.

The results of this research can also have practical application in the education of divers during training. Psychology can offer many useful techniques by which a diver can cognitively restructure negative thoughts and reduce the intensity of the psychophysiological stress response even before the dive and thus prevent the harmful effects of anxiety on diving outcomes. 
Acknowledgments We are very grateful to the study participants, military and recreational divers, who agreed to dive, without any compensation, exclusively for the purposes of this research. Their commitment to diving motivated them to contribute knowledge that can increase diving safety. We also thank the commanders of military professional divers and the leaders of recreational diving clubs who encouraged their members / employees to participate in this study.

Author's contribution All authors contributed to the study conception and design. Material preparation, data collection and analysis were performed by [full name], [full name] and [full name]. The first draft of the manuscript was written by [full name] and all authors commented on previous versions of the manuscript. All authors read and approved the final manuscript.

Availability of data and material (data transparency) The datasets generated during and/or analyzed during the current study are available from the corresponding author on reasonable request.

Code availability (software application or custom code) Not applicable.

\section{Declarations}

Conflicts of interest There is no conflict of interest.

Ethics approval The study was approved by the ethics committee of University of Split, School of Medicine and the study was performed in accordance with the ethical standards as laid down in the 1964 Declaration of Helsinki and its later amendments or comparable ethical standards.

Consent to participate Informed consent was obtained from all individual participants included in the study.

Consent for publication The authors affirm that human research participants provided informed consent for publication of this study.

Conflict of interest The authors have no conflicts of interest to declare that are relevant to the content of this article.

Open Access This article is licensed under a Creative Commons Attribution 4.0 International License, which permits use, sharing, adaptation, distribution and reproduction in any medium or format, as long as you give appropriate credit to the original author(s) and the source, provide a link to the Creative Commons licence, and indicate if changes were made. The images or other third party material in this article are included in the article's Creative Commons licence, unless indicated otherwise in a credit line to the material. If material is not included in the article's Creative Commons licence and your intended use is not permitted by statutory regulation or exceeds the permitted use, you will need to obtain permission directly from the copyright holder. To view a copy of this licence, visit http://creativecommons.org/licenses/by/4.0/.

\section{References}

Anegg, U., Dietmaier, G., Maier, A., Tomaselli, F., Gabor, S., Kallus, K., \& Smolle-Jüttner, F. (2002). Stress-induced hormonal and mood responses in scuba divers: A field study. Life Sciences, 70(23), 2721-2734. https://doi.org/10.1016/S0024-3205(02) 01537-0

Bachrach, A. J., \& Egstrom, G. H. (1987). Stress and performance in diving. .
Beck, A. T. (1980). Cognitive therapy of depression. Wiley.

Beck, A. T. (1991). Cognitive therapy and the emotional disorders. Penguin.

Bentler, P. M. (1990). Comparative fit indexes in structural models. Psychological Bulletin., 107(2), 238-246. https://doi.org/10.1037/ 0033-2909.107.2.238

Bielec, G., Błaszkowska, J., \& Waade, B. (2006). The phenomenon of axiety in scuba divers. Polish Hyperbaric Medicine and Technology, 4(17), 15-20.

Biersner, R. J., \& LaRocco, J. M. (1983). Personality characteristics of US navy divers. Journal of Occupational Psychology, 56(4), 329-334. https://doi.org/10.1111/j.2044-8325.1983.tb00139.x

Biersner, R. J., \& Larocco, J. M. (1987). Personality and demographic variables related to individual responsiveness to diving stress. Undersea Biomedical Research, 14(1), 67-73.

Biersner, R. J., McHugh, W. B., \& Rahe, R. H. (1981). Biochemical variability in a team sports situation. Journal of Human Stress, 7(3), 12-17. https://doi.org/10.1080/0097840x.1981.9936827

Blumenberg, M. A. (1996). Human factors in diving. [Unpublished doctoral dissertation]. Monterey California. Naval Postgraduate School.

Bonnet, A., Fernandez, L., Piolat, A., \& Pedinielli, J. L. (2008). Changes in emotional states before and after risk taking in scuba diving. Journal of Clinical Sport Psychology, 2(1), 25-40. https://doi.org/10.1123/jcsp.2.1.25

Clark, D. M. (1986). A cognitive approach to panic. Behaviour Research and Therapy, 24(4), 461-470. https://doi.org/10.1016/ 0005-7967(86)90011-2

Clark, D. A., \& Beck, A. T. (2011). Cognitive therapy of anxiety disorders: Science and practice. The Guilford Press.

Colodro Plaza, J., Garcés de los Fayos Ruiz, E. J., López García, J. J., \& Colodro Conde, L. (2014). Prediction of human adaptation and performance in underwater environments. Psicothema, 26(3), 336-342. https://doi.org/10.7334/psicothema2014.5

Colvard, D.F. (2000). In: Fathoms of fear: A case study of panic in a recreational scuba diver. Alert diver Asia Pacific, DAN Asia Pacific, pp. 6-10. Retrieved November 20, 2021, from http:// www.danasiapacific.org/main/diving_safety/DAN_Doc/pdfs/ panic2.pdf. (January 2021).

Compare, A., Zarbo, C., Shonin, E., Van Gordon, W., \& Marconi, C. (2014). Emotional regulation and depression: A potential mediator between heart and mind. Cardiovascular Psychiatry and Neurology, 2014, 324374. https://doi.org/10.1155/2014/324374

Dickerson, S. S., \& Kemeny, M. E. (2004). Acute stressors and cortisol responses: A theoretical integration and synthesis of laboratory research. Psychological Bulletin, 130(3), 355-391. https:// doi.org/10.1037/0033-2909.130.3.355

Griffiths, T. J., Steel, D. H., \& Vaccaro, P. (1978). Anxiety levels of beginning scuba students. Perceptual and Motor Skills, 47(1), 312-314. https://doi.org/10.2466/pms.1978.47.1.312

Hammermeister, J., \& Burton, D. (1995). Anxiety and the ironman: Investigating the antecedents and consequences of endurance athletes' State anxiety. The Sport Psychologist, 9, 29-40.

Hanton, S., Neil, R., Mellalieu, S. D., \& Fletcher, D. (2008). Competitive experience and performance status: An investigation into multidimensional anxiety and coping. European Journal of Sport Science, 8(3), 143-152. https://doi.org/10.1080/17461 390801987984

Heyman, S. R., Rose, K. G., Nadeau, C. H., Halliwell, W. R., Newell, K. M., \& Roberts, G. C. (1980). Psychological variables affecting scuba performance. Psychology of Motor Behavior and Sport, 1979, 180-188.

Imber, B. (2018). Integrating stress inoculation training and heart rate variability biofeedback on adult scuba divers in a natural dive setting: A preliminary pre-/posttreatment quasi-experiment 
[doctoral dissertation, Saybrook University] Proquest, https:// www.proquest.com/docview/2267452548/9285D318E3AB471 $6 \mathrm{PQ} / 1$.

Lovibond, P. F., \& Lovibond, S. H. (1995). The structure of negative emotional states: Comparison of the depression anxiety stress scales (DASS) with the Beck depression and anxiety inventories. Behaviour Research and Therapy, 33(3), 335-343. https://doi.org/ 10.1016/0005-7967(94)00075-u

Lucrezi, S., Egi, S. M., Pieri, M., Burman, F., Ozyigit, T., Cialoni, D., Thomas, G., Marroni, A., \& Saayman, M. (2018). Safety priorities and underestimations in recreational scuba diving operations: A European study supporting the implementation of new risk management Programmes. Frontiers in Psychology, 9, 383. https://doi. org/10.3389/fpsyg.2018.00383

Marlinge, M., Coulange, M., Fitzpatrick, R. C., Delacroix, R., Gabarre, A., Lainé, N., Cautela, J., Louge, P., Boussuges, A., Rostain, J. C., Guieu, R., \& Joulia, F. C. (2019). Physiological stress markers during breath-hold diving and SCUBA diving. Physiological Reports, 7(6), e14033. https://doi.org/10.14814/phy2.14033

Mellalieu, S. D., Hanton, S., \& O'Brien, M. (2004). Intensity and direction of competitive anxiety as a function of sport type and experience. Scandinavian Journal of Medicine and Science in Sports, 14(5), 326-334. https://doi.org/10.1111/j.1600-0838. 2004.00389.x

Morgan, M. (1985). Self-monitoring of attained subgoals in private study. Journal of Educational Psychology, 77(6), 623-630. https:// doi.org/10.1037/0022-0663.77.6.623

Morgan, W. P. (1995). Anxiety and panic in recreational scuba divers. Sports Medicine, 20(6), 398-421. https://doi.org/10.2165/00007 256-199520060-00005

Munoz-de-Escalona, E., \& Canas, J. J. (2017). Online measuring of available resources. Paper presented at the H-workload 2017: the first international symposium on human mental work.

Ng, T. W., Sorensen, K. L., Zhang, Y., \& Yim, F. H. (2019). Anger, anxiety, depression, and negative affect: Convergent or divergent? Journal of Vocational Behavior, 110, 186-202. https://doi.org/10. 1016/j.jvb.2018.11.014

Niewiedział, D., Miłkowska, M., Siermontowski, P., Dąbrowiecki, Z., Kaczerska, D., Juszczak, D., \& Olszański, R. (2019). The problem of experiencing anxiety among divers. Experiment in hyperbaric exposure conditions. Polish Hyperbaric Research, 66(1), 47-59. https://doi.org/10.2478/phr-2019-0004

Revelli, L., Addolorato, G., D'Amore, A., Carrozza, C., Giubileo, G., Puiu, A., Lombardi, C. P., Bellantone, R., Gasbarrini, G., \& Progetto Abissi 2005 Dive Medical Group. (2007). Neuroendocrine and psychological assessment in a guinness 10 days scuba dive. International Journal of Sports Medicine, 28(10), 848-852. https://doi.org/10.1055/s-2007-964910

Selection. (2019). The Depression Anxiety Stress Scales. Retrieved August 20, 2021, from http://www2.psy.unsw.edu.au/Groups/ Dass/Croatian/Selekcija\%20DASS/Priru\%C4\%8Dnik\%20DASS\% 20Ivakovi\%C4\%87.pdf.

Siegler, R. S., DeLoache, J. S., \& Eisenberg, N. (2006). How children develop. Worth Publishers.

Spielberger, C. (1989). State-trait anxiety inventory: Bibliography. Consulting Psychologists Press.

Spielberger, C., Gorsuch, R., Lushene, R., Vagg, P., \& Jacobs, G. (1983). Manual for the State-trait anxiety inventory (form Y) mind garden. Palo Alto, CA.

St Leger Dowse, M., Whalley, B., Waterman, M. K., Conway, R. M., \& Smerdon, G. R. (2019). Diving and mental health: The potential benefits and risks from a survey of recreational scuba divers. Diving and Hyperbaric Medicine, 49(4), 291-297. https://doi.org/10. 28920/dhm49.4.291-297

Van Wijk, C. H. (2002). Levels of anxiety and hostility in south African navy divers. Journal of the Undersea and Hyperbaric Medical Society, Inc, 29(4), 271-278.

Van Wijk, C. H. (2014). The use of Spielberger's State-trait personality inventory (trait anxiety subscale) with naval subaquatic specialists. International Journal of Occupational Medicine and Environmental Health, 27(6), 959-966. https://doi.org/10.2478/ s13382-014-0321-5

Van Wijk, C. H. (2018). Personality profiles of divers: Integrating results across studies. International Maritime Health, 69(4), 297-303. https://doi.org/10.5603/imh.2018.0046

Van Wijk, C. H., Martin, J. H., \& Firfirey, N. (2020). Common mental health conditions among navy divers: A brief report. Diving and Hyperbaric Medicine Journal, 50(4), 417-420. https://doi.org/10. 28920/dhm50.4.417-420

Walton, L. (2018). The panic triangle: Onset of panic in scuba divers. Undersea \& hyperbaric medicine. Journal of the Undersea and Hyperbaric Medical Society, 45, 505-509.

Zarezadeh, R., \& Azarbayjani, M. A. (2014). The effect of air scuba dives up to a depth of 30 metres on serum cortisol in male divers. Diving and Hyperbaric Medicine, 44(3), 158-160.

Publisher's Note Springer Nature remains neutral with regard to jurisdictional claims in published maps and institutional affiliations. 